\title{
Educar para la vida y la muerte a los estudiantes de Enfermería: “del miedo al amor"
}

\author{
Educate Nursing students for life and death: "from fear to love"
}

Educar estudantes de Enfermagem para a vida e a morte: "do medo ao amor"

\author{
María Cinta Palomar Gallardo ${ }^{1}$, ORCID 0000-0003-2135-3852 \\ María Romero Palomar ${ }^{2}$, ORCID 0000-0002-5355-9524 \\ Esteban Pérez García ${ }^{3}$, ORCID 0000-0003-1287-0276 \\ María Isabel Romero Palomar ${ }^{4}$, ORCID 0000-0001-5184-0403 \\ ${ }^{1}$ Facultad de Enfermería, Universidad de Huelva. España \\ ${ }^{2}$ Hospital Juan Ramón Jiménez, Huelva. España \\ ${ }^{3}$ Hospital Infanta Elena, Huelva. España \\ ${ }^{4}$ Centro de Salud de Lepe, Huelva. España
}

Recibido: 18/04/2018

Aceptado: $31 / 03 / 2020$

\begin{abstract}
Resumen: Esta investigación surge de la observación fenomenológica como enfermera asistencial cuidando a pacientes al final de la vida y como docente de enfermería en la asignatura "Afrontamiento de la muerte: intervención interdisciplinar". Existen grandes carencias sobre formación en los cuidados al final de la vida, la muerte y el duelo. Para cubrir esta necesidad de formación de los alumno/as realizo una propuesta de intervención docente en la asignatura "Afrontamiento de la muerte: intervención interdisciplinar", cuyo objetivo es evaluar el impacto que la propuesta educativa de la asignatura tiene sobre los estudiantes de Enfermería. Metodología: Esta investigación está enmarcada en el paradigma interpretativo o hermenéutico, de enfoque cualitativo. Desde una mirada fenomenológica, se plantea un estudio descriptivo y como instrumento de investigación se tomarán los diarios reflexivos de los alumnos/as, matriculados en la asignatura que eligieron la opción de asistencia a clase. Resultados: El número de diarios fueron 36, pero se seleccionaron de forma aleatoria 14. El análisis cualitativo ha permitido estructurar el discurso del alumnado en 5 categorías de análisis: Motivo de elección de la asignatura; Crecimiento personal; Logros en el proceso de aprendizaje; Aplicación profesional de las técnicas desarrolladas en la asignatura; Valoración de la asignatura por parte del alumno. Conclusiones: La intervención educativa de la asignatura, ha tenido un alto impacto en los alumnos/as, en dos dimensiones importantes para sus vidas. Por un lado, aumentando sus competencias académicas como futuros profesionales, y por otro adquiriendo habilidades y actitudes positivas sobre la muerte, para vivir y amar la vida.
\end{abstract}


Palabras Clave: educación; muerte; duelo; Enfermería; Terapia Gestalt

\begin{abstract}
This research is based on phenomenological observation both as a nurse caring for patients at the end of life and as a nursing teacher of the subject "Coping with death: an interdisciplinary intervention". The considerable deficiencies in training nurses for care at the end of life, death and grief led me to propose a teaching intervention "Coping with death: an interdisciplinary intervention", the general aim of which was to evaluate the impact of this subject on nursing students. Methodology: this research is framed by the interpretive or hermeneutical paradigm, with a qualitative approach. From a phenomenological point of view, a descriptive study was proposed, and the reflective journals written by students enrolled on the course were used as the research tool. Results: of the 36 journals gathered at the end of the course, 14 were selected randomly. Qualitative analysis enabled the students' discourse to be structured in 5 categories for analysis: reason for choosing the subject; personal development; what I have achieved in the learning process; how to apply the techniques learnt on the course to my professional activity; the students' assessment of the subject. Conclusions: The impact of the educational intervention was high, according to the students, who acknowledged the positive effect on two important dimensions of their lives. On the one hand, it enhanced their academic skills as future nursing professionals, and, they acquired skills and positive attitudes towards coping with death, and how to live and love life.
\end{abstract}

Keywords: education; death; grief; Nursing; Gestalt Therapy

Resumo: Esta pesquisa decorre da observação fenomenológica como enfermeira assistencial que cuida de pacientes no final da vida e professora de enfermagem na disciplina "Enfrentando a morte: intervenção interdisciplinar". Existem grandes deficiências no treinamento de cuidados no final da vida, morte e sofrimento. Para suprir essa necessidade de formação dos alunos, faço uma proposta de intervenção docente na disciplina "Lidando com a morte: intervenção interdisciplinar", cujo objetivo é avaliar o impacto que a proposta educacional da disciplina tem sobre os estudantes de enfermagem. Metodologia: Esta pesquisa está enquadrada no paradigma interpretativo ou hermenêutico, com abordagem qualitativa. Do ponto de vista fenomenológico, propõe-se um estudo descritivo e, como instrumento de pesquisa, serão realizados os diários reflexivos dos alunos, matriculados na disciplina que escolheram a opção de presença nas aulas. Resultados: o número de jornais foi 36 , mas foram selecionados aleatoriamente 14. A análise qualitativa permitiu estruturar o discurso do aluno em 5 categorias de análise: Motivo da escolha do assunto; Crescimento pessoal; Realizações no processo de aprendizagem; Aplicação profissional das técnicas desenvolvidas no assunto; Avaliação do assunto pelo aluno. Conclusões: A intervenção educativa do sujeito teve um alto impacto sobre os alunos, em duas dimensões importantes para sua vida. Por um lado, aumentando suas habilidades acadêmicas como futuros profissionais e, por outro, adquirindo habilidades e atitudes positivas em relação à morte, para viver e amar a vida.

Palavras-chave: educação; morte; pesar; Enfermagem; Terapia Gestalt 


\section{Introducción}

Este trabajo surge de la experiencia personal y profesional. En el año 1990 comencé a trabajar como enfermera en las Unidades Hospitalarias de Medicina Interna, donde se atendía pacientes con patologías terminales (Cáncer, Sida, Enfisemas Pulmonares) y en los que se incluían los Cuidados Paliativos. Estos se basan en la asistencia total y activa a los pacientes y a su entorno por un equipo interdisciplinar cuando no hay esperanza de curación y cuyo objetivo esencial del tratamiento ya no consiste en prolongar la vida sino en asegurar la máxima calidad de vida posible, tanto para el paciente como para su familia. Comprenden la atención de los aspectos físicos, emocionales, sociales y espirituales, promocionando la dignidad y autonomía de los enfermos y su familia, extendiéndose hasta el duelo. No prolongar la vida y tampoco aceleran la muerte, solamente intentar estar presentes y aportar los conocimientos especializados que requieren estos pacientes $(1,2,3,4)$. Para realizar estos cuidados, estaba cualificada técnicamente pero no psicológicamente, teniendo que afrontar la muerte de los pacientes y los duelos de los familiares. Este fue el motivo que me llevó a buscar apoyo e instrumentos necesarios para poder acompañar como enfermera a los pacientes y a su familia al final de la vida, muerte y duelo.

Posteriormente en el año 2000, accedo a la facultad de Enfermería de la Universidad de Huelva como profesora titular. Desde este nuevo lugar de trabajo y por todas las vivencias compartidas con estos pacientes y sus familiares en dichas unidades hospitalarias, presenté una propuesta docente en mi facultad donde quedaba justificada la necesidad de formar a los futuros profesionales de enfermería en Cuidados Paliativos. (4) En el 2001, se comenzó a impartir la asignatura de Cuidados Paliativos como asignatura optativa en la Facultad de Enfermería de Huelva $(5,6,7)$. Esta asignatura está incluida dentro del plan de estudio de la carrera de grado de enfermería como obligatoria.

En el año 2011, presente otra una nueva propuesta docente que fue aprobada por Facultad de Enfermería de la Universidad de Huelva, la asignatura de "Afrontamiento de la muerte: intervención interdisciplinar. Justificando la necesidad de ampliar las horas para abordar los cuidados al final de la vida, muerte y duelo. Esta asignatura me ha dado la oportunidad de profundizar más con los alumnos/as de enfermería, por lo que he elegido esta asignatura para desarrollar la investigación de este trabajo.

Como docente, mi gran preocupación y mis preguntas constantes siempre fueron enfocadas a:

¿De qué manera puedo yo hacer que los alumnos/as tomen conciencia de que los Cuidados Paliativos o los cuidados al final de la vida muerte y duelo, les van a aportar un gran crecimiento personal y profesional?;

¿Cómo puedo cambiar sus miradas de miedos y rechazos a estos pacientes, por una mirada de amor y respeto hacia ellos y sus familias?;

¿Cómo les puedo enseñar que los que van a morir nos enseñan a vivir?;

¿De qué forma puedo decirles a ellos que tomar conciencia de muerte es tomar conciencia de vida?, es vivir y amar la vida. 
Todas estas preguntas fueron encontrando algunas repuestas a través de talleres de formación en distintas técnicas de la psicoterapia, destacando mi formación en Pedagogía Sistémica $(8,9)$ y en la Psicología de los Sistemas con el enfoque de Bert Hellinger $(10,11)$. A nivel personal y profesional me ha aportado una forma diferente de pensar y de disfrutar de labor de educar sobre los cuidados al final de la vida, muerte y duelo, desde el agradecimiento y respeto profundo a la vida tal cual es, tal cual soy yo, tal cual son los demás y tal cual son mis padres (12).

Las actitudes y comportamientos del ser humano ante la muerte son aprendidos culturalmente. Dichas costumbres han variado de un tiempo a otro y con ello la concepción del concepto de muerte, pasando de ser un hecho natural e inevitable a considerarse un enemigo al que hay que conquistar $(3,14)$. Cuando los recursos terapéuticos se agotan para estos pacientes, son muchas las enfermeras/os que creen, "que ya no se puede hacer nada más por estos pacientes" (15) El problema que he percibido en estos profesionales es que no saben qué hacer o decir, en esos momentos, les falta formación e instrumentos para enfrentarse al proceso de la muerte y el duelo. $(15,16,17)$.

Esta investigación surge de la observación fenomenológica de la experiencia profesional como enfermera asistencial cuidando a estos pacientes y como docente con los estudiantes de enfermería. Evidenciando como ambos tienen grandes deficiencias de formación sobre los cuidados al final de la vida, la muerte y el duelo (18). Para cubrir esta necesidad de formación a los alumno/as de enfermería, realizo una propuesta de intervención docente en la asignatura "Afrontamiento de la muerte: intervención interdisciplinar".

\section{-Objetivo General}

Evaluar el impacto que la propuesta docente de la asignatura "Afrontamiento a la muerte: intervención interdisciplinar" tiene sobre los estudiantes de Enfermería.

-Objetivos específicos:

- Formar a los estudiantes sobre los conocimientos de los Cuidados al final de la vida, la muerte y el duelo.

- Concienciar a los estudiantes sobre la importancia de los cuidados al final de la vida, la muerte y el duelo para su crecimiento profesional y personal.

- Modificar la actitud de rechazo de los estudiantes en el proceso de la muerte y el duelo, para conseguir unos Cuidados de Calidad.

- Ayudar a los alumnos/as a superar los miedos y temores hacia la muerte.

- Aceptar la muerte como parte de la vida, agradeciendo todo lo aprendido en el proceso de la muerte y el duelo

- Integrar en la filosofía de vida de los alumnos/as que "ayudar a morir implica aprender a vivir" y que "tomar conciencia de muerte es tomar conciencia de vida".

Los futuros profesionales de enfermería deben ser capaces de superar los miedos y temores hacia la muerte, deben de tomar conciencia de que la muerte forma parte de la vida y hay que saber estar ahí, acompañando al paciente, mirándolo a los ojos sin miedos, desde el amor, desde un profundo agradecimiento hacia al paciente, hacia su familia y a la vida, por todo lo que nos dio y nos enseñó durante su proceso. Cuando profundizamos en la filosofía Paliativa, Sistémica, Gestalt somos capaces de cambiar esa mirada de miedo a la muerte del paciente por otra mirada de amor 
y aceptación hacia él, asintiendo lo que en ese momento toca vivir y en la que la enfermera como ayudadora, acompaña desde el respeto y la humildad.

\section{Metodología}

Esta investigación está enmarcada en el paradigma interpretativo o hermenéutico. El enfoque cualitativo que se deriva de este paradigma nos permite mirar desde una perspectiva holística $(24,25)$. Coincidiendo con los valores tradicionales aceptados en la enfermería. $(26,27,28)$.

Desde una mirada cualitativa, se plantea como instrumento de investigación los diarios reflexivos de los alumnos/as. La población de estudio, serán todos los alumnos/as matriculados en la asignatura de "Afrontamiento a la muerte: intervención interdisciplinar" en el curso académico 2014/2015 de la facultad de Enfermería de la Universidad de Huelva, que eligieron voluntariamente la opción de asistencia a clase. En total fueron 36 alumnos los que eligieron dicha opción. A todos ellos se les explico detalladamente como se llevaría a cabo la realización de la intervención docente y como, a través de sus diarios personales, recogeríamos la información de dicha investigación.

La metodología utilizada en la asignatura requiere de una serie de actividades y estrategias didácticas. Las bases conceptuales de estas actividades se encuentran ubicadas dentro de la Psicología Humanista, concretamente entre la Psicoterapia Gestáltica y Sistémica.

Las herramientas para estos talleres serían entre otros: los movimientos sistémicos, el dibujo, el genograma familiar, la musicoterapia, la bioenergética, la dramatización y las vivencias personales. A la finalización de cada sesión, los alumnos/as tendrán unos 20 minutos aproximadamente, para que escriban en sus diarios sus experiencias de ese día de clase. El diario reflexivo pretende que el alumno/a contemple e integre la información de la experiencia de enseñanza-aprendizaje vivida $\mathrm{y}$, comunique de forma escrita $\mathrm{y}$ libre, su percepción de la experiencia, sentimientos, emociones, pensamientos, ideas, reacciones y afrontamientos. Este tipo de diarios, le permite al estudiante alcanzar mayor nivel de reflexión, que le llevará al aprendizaje transformativo $(29,30)$.

Tomamos como marco referencial la fenomenología, ya que los talleres teóricovivenciales, fueron focalizados en la experiencia individual de cada uno de los alumnos/as. Es decir, que la experiencia personal de la muestra (alumnos/as junto a la profesora) será el hilo conductor de este trabajo de investigación cualitativa, en el que se pretende evaluar el impacto que la propuesta educativa de la asignatura tiene sobre los estudiantes de enfermería, para obtener los resultados y poder realizar las conclusiones.

\section{Resultados}

El número de diarios fueron 36, pero se seleccionaron de forma aleatoria 14 por saturación de información. El análisis cualitativo ha permitido estructurar el discurso del alumnado en 5 categorías de análisis. La primera categoría "Motivo de elección de la asignatura" recoge las razones de la elección de la asignatura; en la segunda categoría "Crecimiento personal" se analizan la evolución de la actitud del alumnado en relación al proceso de la vida y la muerte; la 
tercera categoría "Logros en el proceso de aprendizaje”, describe y analiza los conocimientos y habilidades adquiridas durante la intervención docente; cuarta categoría, "diferentes vías de aplicación profesional de las técnicas desarrolladas durante la intervención docente" $\mathrm{y}$, en la quinta categoría "Valoración de la asignatura por parte del alumno" se exponen las opiniones vertidas así como la autoevaluación de la asignatura.

Categoría 1. Motivos de elección. En esta categoría se expresa el interés de los alumnos/as, por aumentar sus conocimientos sobre el proceso de morir y su crecimiento personal.

"...me ha ofrecido una segunda oportunidad para poder formar parte de clases tan impresionantes como lo es afrontamiento a la muerte, donde en definitiva aprendemos a vivir en vez de sobrevivir” ...d14p3

Categoría 2. Crecimiento personal. La información que emerge en esta categoría se ha estructurado en cuatro sub-categorías, que se han denominado: Autoestima, Identidad, Toma de Conciencia y Relaciones entre Participantes.

- Autoestima. En este apartado sobre la autoestima, se ha recogido aspectos, sobre el sentir de los alumno/as de su valía personal.

"Escuchando a mis compañeros sentía mucha fuerza, alegría ganas de vivir, de disfrutar de la vida, de los míos; fue una experiencia con unas sensaciones muy bonitas” ... d2p3

- Identidad. Dentro de esta subcategoría sobre la identidad se han recogido valores, tradiciones, creencias, comportamientos a nivel individual y de grupo

"Algunas de las palabras que obtuve fueron bienestar, diversión, paz, raíces, alegría, libertad, amor, ilusión, esperanza, armonía, serenidad, humildad, cosas que forman parte de mí y que me hacen sentir viva" ...d14p9.

- Toma de conciencia. El desarrollo de los contenidos y el planteamiento de determinadas actividades como la del "ciclo vital" han ayudado al alumnado a una nueva toma de conciencia y desarrollo personal.

"En cada una de ellas fuimos imaginando lo que nos ocurrió u ocurrirá en un futuro a lo largo de nuestra vida, por lo que fue una actividad fructifera y llena de sentimientos a flor de piel" ...d30p6

Hay que destacar en la segunda evidencia, como en el desarrollo de esta actividad supone un acto de humildad. El recorrido por las diferentes etapas de sus vidas, una vez llegado a la vida propiamente dicha, les hace reconocer la grandeza de la vida.

“...al llegar a la vida me di cuenta que tenía que bajar la cabeza y no enfrentarme a la vida sino dejarme fluir con ella”...d29p2

Este acto de humidad ante la vida, se reforzó en muchos momentos vividos con ellos en las clases. Sobre la toma de conciencia de sus raíces. Como podemos, ver, emergen expresiones muy emotivas como la "madre tierra", "donde nacimos"," vivimos", "es algo muy grande" ... Todas estas expresiones de amor, de vínculo y profundo respeto a su tierra se escucharon cuando se realizaba el taller de "La madre tierra". Como muy bien se refleja en esta por las evidencias, muchos/as de los/as alumnos/as no son de Huelva. Ellos/as se sienten acogidos/as y agradecidos/as, pero echan de menos su tierra, su raíz, su fuerza. 
“...es algo muy grande, en la que estamos inmersos en nuestro día a día. La madre tierra donde nacimos y vivimos es lo más grande que podemos tener ya que en ella se sumerge también todos nuestros seres queridos que nos rodean” ...d30p4

Se observan evidencias de afrontamiento personal eficaz ante la vida y la muerte, las evidencias recogidas han sido dos, en la primera ante la vida se recoge, un manifiesto contundente, claro, conciso donde se expresa, que todo lo que necesitamos para triunfar en la vida, está dentro de nosotros.

"Tener claro que todos somos únicos, completos 100\%, que albergamos en nuestro interior todo lo que necesitamos para triunfar en esta vida" ... d6p4

Y, por otro lado, la aceptación de la muerte como parte natural de la vida. Reflexionando sobre estas evidencias, nos conmueven las manifestaciones de unos de los alumnos/as, sobre la vida y la muerte:

"Ahora lo único que me preocupa es vivir porque la muerte como ya sabemos forma parte de la vida y llegara cuando tenga que llegar, pero de lo que estoy segura es de que cuando llegue habré vivido, respirado, sentido, como yo he aprovechado cada momento" ...d16p5

Respecto a la Toma de conciencia del sistema familiar, quisiera resaltar tres aspectos porque en cada uno de ellos surgen matices diferentes que a continuación explico. En la primera evidencia se manifiestan expresiones de miedo del sistema familiar, "a no controlar" "a que cambie algo" "miedos" que hasta que no terminaron no fueron conscientes de ellos.

"Cuando terminé, fui consciente de todo lo que había reflejado en la cartulina. Miedo al cambio, al ritmo de las personas de mi alrededor, miedo a no controlar el tiempo y las situaciones cambiantes constantemente” ...d6p11-12

En la segunda evidencia se manifiesta, la toma de conciencia de que en su sistema familiar, hay un problema de relación con su madre, "No escucha a su mamá" lo soluciona hablando con su mamá "ambas nos sentimos mejor.

"...hablando con mi madre, se desahogó conmigo ...hablamos de cosas que tenemos en común... yo nunca me había dado cuenta por no escucharla, después de esto ambas nos sentimos mejor" ... d30p2

En la tercera evidencia se refleja como el alumno/a a través de sus palabras manifiesta que se siente "algo desvinculada del círculo familiar. Las tres evidencias fueron fruto del trabajo realizado en la actividad "sistemas familiares y miedos".

"Mientras, mis hermanas están muy unidas a mi madre y la apoyan, pero yo tengo que hacerlo desde la distancia al estar estudiando fuera, y eso hace que me sienta algo desvinculada del circulo" ...d14.p6

Respecto a la toma de conciencia de nuestros "papas niños." Observamos a partir de sus manifestaciones la toma de conciencia de los alumnos/as con respecto a sus padres, reconociendo que está vinculado a su sistema familiar, pero también a otros sistemas. "Me hizo ver que nuestros padres, no son sólo padres, son hermanos de otros, amigos de otros, nietos de otros, hijos de otros" ... $d 18 p 6$

Por otro lado, los estudiantes expresan emoción al visualizar la "mirada inocente" de sus padres cuando fueron niños.

"Me senti bastante emocionada, nunca me habia parado a pensar que alguna vez habian sido niños también, y pude ver esa mirada de inocencia que algún día tuvieron” ...d14p 7 
Estas manifestaciones surgen cuando se realizó a través de fotos de sus padres la actividad "Nuestros padres niños".

- Relaciones entre participantes. Como podemos ver en las evidencias primera y segunda. El alumnado expresa sentimientos de felicidad por las palabras de sus compañeros/as hacia ellos/as. En la evidencia, tres alumnos/as expresan su satisfacción por las palabras de sus compañeros y, además, afloran sentimientos de agradecimiento hacia sus compañeros "los tendré a mi lado para toda mi vida". Estos sentimientos de felicidad y agradecimientos a los compañeros por sus bonitas palabras, se vivieron en la actividad "mi casa" favoreciendo las relaciones entre los participantes.

"Esta dinámica, me sirvió para escuchar palabras bellas de mis compañeros sobre mí, ya que, en la rutina, no encontramos momentos para ello, y me sentí como que estos cuatro años habían merecido la pena junto a ellos, y que los tendré a mi lado para toda la vida”...d18p8

Otra evidencia de este apartado expresa la unión del grupo de alumnos-as. Se expresa con palabras de cariño, apoyo y compresión:

"La satisfacción de haber logrado decir lo que nunca pude, reforzando "el sí puedo" ... "he podido decir cosas que llevo tiempo queriendo decir y nunca he encontrado el momento" $d 4 p 8$

Estas evidencias corresponden, al igual que las anteriores, a la actividad "mi casa", que se realizó con el objetivo de reforzar las relaciones en el grupo. Esto supone una estrategia básica para la cohesión del grupo de alumnos y fundamental para abordar contenidos tan delicados y profundos, que difícilmente se podrían tratar de otra forma.

Dentro de las relaciones, se consigue mediante la actividad "sanando nuestras relaciones," que el alumnado sane sus relaciones. En este sentido emergen sentimientos contrarios. Por una parte, expresan liberación al sanar las relaciones, como podemos ver en las evidencias primera

"Gracias al trabajo... me he liberado y he podido resolver un problema que por no hablar y comunicar... cada vez se hacía más grande”...d16p6.

Mientras que la segunda evidencia expresa me "desahogue" y "note pasotismo", no saneando las relaciones.

“...mediante una actividad que nos permitió ponernos en el lugar del otro. En mi caso me desahogué con mi padre con el que no mantengo una buena relación y por su parte note pasotismo y agobio" ...d14p8

En esta actividad, se pudo observar, como algunos pudieron sanear sus relaciones por los gestos de sus caras, sus posturas, sus miradas y otros no.

Es importante para las relaciones desde el enfoque sistémico el equilibrio entre el dar y recibir fue, "El túnel de las caricias".

Al igual que la anterior subcategoría comentada las evidencias se contradicen. En la primera evidencia expresan haberse sentido "estupendamente" se dejaba recibir las caricias, abrazos con agradecimiento a los compañeros

"me he sentido estupendamente cuando mis compañeros me demostraban su cariño al paso del túnel”... d16p3

Sin embargo, en la segunda evidencia, todo lo contrario, le cuesta recibir esas caricias y abrazos; lo expresa diciendo 
"me cuesta que atraviesen mi espacio vital". "Al igual en la vida, en este no me he sentido cómoda. Soy cariñosa con muy pocas personas, fría con el resto y me cuesta que atraviesen mi espacio vital" ...D6p12

Estas dos evidencias encontradas se observan con mucha frecuencia cuando se realiza esta actividad; la resistencia de algunos alumnos/as a recibir caricias y abrazos. Esta dificultad tiene un trasfondo sistémico, las lealtades a nuestro sistema.

Categoría 3. Logros en el proceso de aprendizaje. Desarrollada en las siguientes subcategorías: Conocimientos: sistémica; Conocimientos: muerte-vida; Habilidades; Conocimientos Duelos; Actitud y Conocimientos: Terapia psicocorporal.

- Conocimientos: sistémica. En esta categoría, como podemos ver por las evidencias recogidas, los alumnos y alumnas manifiestan y reflexionan sobre sus conocimientos de la mirada sistémica y los órdenes del amor.

"Todo esto nos hace pararnos a reflexionar sobre si realmente nuestro entorno constituye un sistema y si realmente funciona como tal o no. Es sorprendente lo que podemos descubrir y entender después de esto" ...d14p8

"La importancia de equilibrio entre el dar y recibir para que una relación sea sana" $\ldots d 29 p 1$

Lo que se recoge en ambas evidencias es que los alumnos reconocen la importancia de su lugar en su sistema y el equilibrio entre el dar y recibir. Estos conocimientos fueron adquiridos en la clase teórica de "Pedagogía Sistémica" reforzándose estos conocimientos con otras actividades.

En las siguientes evidencias, a través de sus comentarios, se manifiesta el conocimiento sobre los "ordenes de la ayuda".

Reflexionando sobre estas evidencias, los alumnos/as reconocen que cada persona tiene fuerza para afrontar sus diferentes procesos de vida y que ocupando el lugar del otro a éste lo hacemos débil.

“... tengo que trabajar sobre esto ya que si ocupo el lugar de mi padre, por ejemplo, a él lo hago pequeño, como si él no fuera capaz de hacerlo, fomentando la dependencia".. d16p4

- Conocimientos: muerte-vida. Como podemos apreciar en las evidencias recogidas en esta categoría, las manifestaciones son contundentes con respecto a cómo hay que afrontar la vida sin juicios previos:

"Ha sido por tanto una clase reflexiva, donde hemos tomado consciencia de la realidad que nos rodea y donde una vez más, hemos podido ver que la muerte forma parte de la vida y que, por tanto, debemos afrontarla como tal”...d30p 12

En la siguiente evidencia, se manifiesta claramente la diferencia que existe entre dolor y sufrimiento, temáticas tratadas en las clases teórica de la asignatura.

"El dolor en el presente, ya que somos protagonistas del presente, en el momento que vivimos recordando el pasado como angustiándonos por un futuro incierto, no presentamos dolor, sino sufrimiento, que no es más que el dolor toxico” ... d6p6

En otra evidencia de esta categoría destacan dos aspectos importantes sobre el tema de cómo tratar al paciente, como persona, como adulto y darle la oportunidad de cerrar sus temas pendientes. 
"La necesidad de la persona moribunda a ser tratado como es, una persona con todos sus sentimientos y emociones, que lo sepan escuchar y comprender por lo que está pasando, que no se le trate con miedo y se le dé la oportunidad de cerrar sus temas pendientes, así como recordarle todo lo bueno pasado" ... d9p6

En esta evidencia se refleja la importancia de que la familia deje ir al paciente con amor, aceptando que llegó el final de su vida.

"Dejar ir a los seres queridos desde el agradecido recuerdo, desde el amor. Por lo tanto, desde el amor, hay que dejar ir a las personas cuando es su momento, y entender, que su vida debe acabar" ... d18p10

Continuando con la misma categoría, este apartado, está dedicado al conocimiento de cómo los profesionales podemos acercarnos a las necesidades espirituales de forma individual, acompañándolos y respetándoles en todo momento sus decisiones.

“...me ha abierto los ojos y podemos acercarnos a las necesidades que éstos, les gustarían que se les cubrieran en los últimos días de su vida” ...d18p10

Igualmente se manifiesta, con rotundidad, que todas las muertes son únicas y únicas es la forma de acompañarlas

"única es la manera de morir de cada una". d4p2

En la última evidencia de esta categoría, podemos ver expresiones de deseo de vivir la vida desde la conciencia de vivirla con plenitud, agradecimiento, para cuando llegue el final de su vida, reconocer que consiguió vivirla como realmente quería.

"Debemos vivir con plenitud para que cuando llegue este momento final y miremos atrás nos sintamos bien con nosotros mismos, dando gracias a la vida por lo conseguido” ...14p10

La toma de conciencia sobre la relevancia de la motivación en la vida, para darle sentido a la vida y alcanzar sus metas, las encontramos en expresiones como.

"La motivación para seguir dándole sentido a nuestra vida y siguiendo nuestra meta de la vida” ...d2p5

Reflexionando sobre estas dos evidencias, nos damos cuenta de la evolución o el cambio de mirada que ha tenido el alumnado respecto a la vida tras la intervención docente. En la mayoría de las clases impartidas, la reflexión sobre la frase "tomar conciencia de muerte es tomar conciencia de vida", les ha calado profundamente.

- Habilidades. En este apartado, se recogen manifestación de satisfacción por la actividad denominada "mirando al otro y sintiendo".

"Fue sorprendente ver como en un 90\% de los casos acertamos en los sentimientos del compañero. En mi caso podía sentir como, a pesar de verse como una chica destacada y echada para adelante, tenía temor en su mirada”...d14p7

- Conocimientos: duelos. Respecto a conocimiento sobre el duelo, en sus evidencias observamos los conocimientos adquiridos sobre este tema.

Encontramos una serie de objetivos que nos ayudarán a resolver el duelo de una forma óptima” ...d30p14

En la segunda evidencia se recoge el sentimiento por el que se determinara si un duelo está resuelto o no... 
"El agradecido recuerdo, es un sentimiento reparador que nos da la clave del saneamiento total de nuestra culpa. Es la señal adecuada que nos indica la resolución completa de nuestro duelo" .... d30p14-15

Continuando con esta categoría, la evidencia recogidas nos manifiesta que las pérdidas no solo están relacionadas con la muerte, sino que existen otros tipos de pérdidas que conducen también a tener que realizar la elaboración del proceso del duelo.

"Tipos de pérdidas"... "Hemos podido observar cómo no todas las pérdidas son enfocadas a la muerte de una persona, sino que también encontramos duelos afectivos, corporales, sociales y evolutivos" ...p30p14

En este último apartado sobre conocimiento del duelo, se pone de relieve el trabajo que se realiza en el proceso de elaboración del duelo, en momentos diferentes del mismo.

En la primera evidencia se expresa con entusiasmo y cariño los logros conseguidos por su compañero y como este se entregó en la dinámica.

"Mi compañero trabajó la pérdida de su abuelo, y expresó todo lo que le querría decir si pudiera verlo por última vez, y después sintió lo que su abuelo lo quería y lo orgulloso que estaba de él. Fue emocionante verlo, ya que veía a mi compañero realmente afectado y entregado a la dinámica, una dinámica fuerte emocionalmente”...d18p12

Estas evidencias corresponden a la teoría del duelo y a la actividad "trabajando el duelo".

Otra de las evidencias que se recoge es el sentimiento de agradecimiento. Se expresan llegando a aumentar la autoestima de la persona "quererte a ti misma".

"Gracias por todo lo que he pasado porque esto te ha hecho mucho más fuerte y te ha mostrado lo bella que es la vida... lo más importante quererte a ti misma como nunca te has querido. Porque para ver el arcoíris tienes que soportar la lluvia”... D29p4

Otra evidencia a resaltar recogida son la manifestada sobre el duelo y su crecimiento personal, manifiesta la importancia del trabajo del duelo a nivel profesional y reconociendo el aprendizaje personal de este.

"Es importante, tanto a nivel personal como profesional, el trabajo de las pérdidas ya que nuestra vida se sume en multitud de pérdidas de las cuales debemos saber salir engrandeciéndonos y aprendiendo de ellas haciéndonos más fuertes " ...d16p6

Por otro lado, resalta el nivel madurativo de la persona, al aceptar la rotura de su relación recordando de esta, sus buenos momentos vivido con ella.

“...Esta dinámica me ayudó, puesto que traté una relación que perdí con una de mis mejores amigas... dejarla a un lado en mi vida y recordar sólo los buenos momentos vividos”...d18p8

- $\underline{\text { Actitud. }}$. En esta subcategoría se refleja un agradecimiento profundo hacia sus seres queridos que han fallecido y un gran respeto para todos los desaparecidos. La expresión en esta evidencia es de amor, orgullo y agradecimiento por todo los que les aporto en su vida. Estas manifestaciones se vivieron en la actividad "El día de los difuntos".

"Le hemos dado las gracias a nuestros fallecidos con bonitas palabras y sintiéndonos orgullosos de todo lo que aportaron a nuestras vidas...un lugar en nuestro corazón a todos aquellos desaparecidos" ... d18p5

- Conocimiento: Terapia Psico-corporal. En esta subcategoría, se recoge evidencias de los conocimientos sobre la Bioenergética (terapia psico-corporal). Se expresa la importancia de esta 
para liberar sus tensiones y escuchar su cuerpo. Así como la toma de conciencia de sus bloqueos energéticos y el reconocimiento de "que algo tiene que hacer"...

"liberando tensiones y emociones, y escuchando un poco nuestro cuerpo. ..., pude ver mis bloqueos que no me dejaban expresarme de la mejor forma y que me hicieron reflexionar sobre qué debía hacer" ...d14p3

Estas expresiones son de los talleres que se realizaron de bioenergética.

Otra evidencia importante que resaltar de esta terapia-psicorporal es el grado de bienestar que les produce el recibir el masaje bioenergético...

"Ha sido maravilloso sentir el calor de las manos en la espalda, como te liberas de todas las presiones”...d29p1.

Categoría 4. Aplicación profesional de las técnicas desarrolladas en la asignatura. La información que emerge en esta categoría se ha estructurado en cuatro subcategorías:

- Actitud del enfermero/a ante la muerte. Observamos sobre esta subcategoría, frases de gran calado en el alumnado, sobre las clases teóricas centrada en "las cuatro tareas para de la vida y la muerte". Sus expresiones son realizadas desde el corazón, no de la razón o intelecto. Estas expresiones nos indican la satisfacción personal de saber estar ahí acompañando al paciente al final de sus días, como personas y como profesionales de los cuidados.

“...me ha enseñado que es lo que estos seres de luz quieren en sus últimos días de vida, como enfermera me propongo mirarlo a los ojos y verlo como una persona fuerte y mirarlo desde el amor" ... d29p4

- La mirada sistémica en los cuidados de enfermería. En esta subcategoría, la evidencia expresada, han trasferido los conocimientos del enfoque sistémico a los cuidados de enfermería, reconociendo que detrás del paciente hay un sistema familiar y una historia que mirar. Se manifiesta también en esta evidencia el enfoque de la mirada sistémica, sobre los Órdenes del Amor. Donde se recoge el tercer Orden del Amor, ocupar el lugar que a cada uno le corresponde.

"... apreciarlo tal cual es dentro de su sistema y saber quedarnos en nuestro lugar en todo momento. Toda persona adulta tiene su propia historia de vida... enfermo terminal "... d6p21

- El valor de la empatía en los cuidados enfermero. Analizando las evidencias del valor de la empatía, se expresa el calado o el valor tan profundo que se le da a la empatía en los cuidados de enfermería durante toda la titulación.

La empatía es un valor que cada uno de los profesionales sanitarios y en especial, nuestra profesión, debe adquirir desde el principio ya que es una competencia fundamental a la hora de desarrollarnos y crecer como buenos profesionales, así como personas "...d30p10.

Valorando la siguiente evidencia, se manifiesta la aptitud de respeto, apoyo y aceptación hacia el paciente.

"Como enfermeras, debemos permanecer a su lado, respetar sus decisiones, apoyarlo, escuchar todos sus miedos, juicios y dolencias sin juzgarlo” ...d18p10.

Reflexionando sobre estas evidencias, consideramos que son de la teoría de las necesidades del moribundo y también de la actividad "saneando nuestras relaciones" ya que se concluyó esta actividad reflexionado y debatiendo en el grupo sobre el ser humano, en el entendido que el mismo es un ser completo cuando acepta tanto su parte positiva como la negativa. 
- El profesional, cuidarse para cuidar. La primera evidencia de esta subcategoría aporta, por sus expresiones, el trabajo personal de crecimiento realizado por el alumnado en la asignatura. Una de las frases que ha resonado bastante en clase ha sido "el cuidado a uno mismo, precede al cuidado hacia los demás", reforzándose en la actividad de bioenergética. En ella como se expresa en la primera evidencia, "expulsar lo malo y quedar lo bueno" en esta técnica se les enseña a inspirar lo bueno que te ha dado la vida ese día y a expirar lo que ahora, en este momento, no te gusta de la vida.

“... expulsar lo malo que nos invade y quedarnos de esa forma con lo bueno y productivo de nuestro día a día y de nuestra vida en general, de esta forma ayudamos a darnos fuerza los unos a los otros y podemos llegar, así, a ser mejores profesionales con nuestros pacientes, en momentos duros y no tan duros" ... d30p3

Categoría 5. Valoración de la asignatura por parte del alumnado. La categoría "valoración de la asignatura por parte del alumnado" expresan sentimientos de gratitud por la misma. También expresas las evidencias recogidas que esta asignatura les ha aportado, conocimiento y habilidades para ser mejor profesional y persona. La reflexión de estas evidencias es fruto de su proceso madurativo o evolutivo durante la intervención docente. Reconocen haber adquirido competencias enfermera para abordar el proceso de morir y su propio proceso de vida.

"Tratar con la muerte es algo que caracteriza a nuestra profesión, y en definitiva a la vida. Para abordarla satisfactoriamente tanto a nivel personal como profesional y afrontarla debemos obtener una serie de competencias imprescindibles, competencias que esta asignatura me ha aportado. La he vivido y sentido llegando a pasar momentos duros. Me fascina este tema y quiero seguir preparándome para no solo ser una buena enfermera de la vida, sino también de la muerte" ... $d 14 p 15$

"Me parece una asignatura tan bella y tan real, te agradezco que nos trasmitas esa necesidad que tú ves de conocernos a nosotros mismos y a nuestros miedos y como llevar a las personas en sus últimos momentos de su vida” ...d29p5

\section{Discusión}

Dada la novedad y originalidad de esta investigación, no he encontrado referencias bibliográficas sobre este tema, por lo que los resultados obtenido de nuestra investigación y conclusiones no podrán ser contrastados y discutidos con otros estudios. Pero si podemos describir en un breve resumen lo que ha significado los resultados obtenidos en las evidencias recogidas en los diarios analizados. Los alumnos/as expresan sentimientos de gratitud hacia la asignatura "afrontamiento a la muerte: intervención interdisciplinar", expresando que les ha aportado, confianza a nivel personal y profesional y reconocen haber adquirido competencias enfermeras para abordar el proceso de morir y su propio proceso de vida. Los alumnos/as sienten y expresan el deseo de vivir la vida desde la conciencia de vivirla. Descubrieron que son capaces de acercase como profesionales a las necesidades espirituales del paciente, también manifiestan el cambio o evolución personal y la fuerza que les ha dado la asignatura para aceptar su responsabilidad y respetar su lugar en su sistema familiar, para sentirse libres. 
Propuestas de mejoras. Consideramos muy importante abrir nuevas líneas de investigación sobre la aplicación de la mirada Sistémica y Gestáltica en la formación a final de la vida muerte y duelo de los futuros profesionales de enfermería:

- La inclusión de la Psicología Humanista, concretamente la Psicoterapia Gestáltica y Sistémica en la formación los futuros profesionales de enfermería.

- Formar a los estudiantes y profesionales de enfermería desde la mirada sistemática, donde se incluya los Órdenes del Amor y los Órdenes de la Ayuda en su quehacer diario dentro de los cuidados de enfermería.

\section{Conclusiones}

A continuación, pasamos a responder a cada uno de nuestros objetivos específicos planteados para la intervención docente, para al finalizar responder al objetivo general.

\section{Objetivos específicos:}

- Realizar un puente teórico y conceptual entre los principios filosóficos de los Cuidados Paliativos y la Mirada Sistémica.

Los alumnos/as integran las dos disciplinas, durante el proceso enseñanza- aprendizaje.

-Dar a conocer a los alumnos/as la mirada sistémica para mejorar los Cuidados de Enfermería al final de la vida, la muerte y el duelo.

Los conocimientos y habilidades adquiridos por los alumnos/as durante la intervención educativa, con la inclusión de la mirada sistémica, ha contribuido a mejorar los cuidados de enfermería al final de la vida, muerte y duelo.

-Desarrollar en los estudiantes un cambio de actitud positivo ante el proceso de la muerte y el duelo.

La intervención de la propuesta docente en los alumnos/as, ha favorecido un cambio positivo ante el proceso de la muerte, duelo y hacia la vida.

-Formar a los alumnos/as en técnicas de Psicoterapia Humanista para favorecer un afrontamiento eficaz ante el proceso de muerte y duelo.

Todas las técnicas de Psicoterapia Humanista utilizadas en la intervención docente para favorecer un afrontamiento eficaz a los alumnos/as ante el proceso de muerte y duelo, fueron determinantes y necesarias para conseguir este objetivo.

-Despertar en el alumno/a interés sobre el proceso de morir y duelo y su implicación con la mirada sistémica.

Se consiguió con la intervención docente, aumentar el interés de los alumnos/as al incorporar esta mirada sistémica en el proceso enseñanza- aprendizaje.

-Conseguir que el alumno/a tome conciencia de la importancia de la vivencia del proceso de la enfermedad terminal, muerte y duelo para su crecimiento personal y profesional.

En el trascurso del tiempo de la intervención docente, los alumnos/as han tomado conciencia de la importancia de acompañar y vivir este proceso, tanto para su crecimiento personal como profesional.

-Mostrar al alumno/a el agradecimiento y la admiración como valores para poder aprender. Este objetivo de agradecimiento y admiración como valores para aprender, se ha cumplido con la intervención educativa en el transcurso de dicha intervención docente. 
-Incluir a los alumnos/as los órdenes de la ayuda en el quehacer diario como profesionales de los Cuidados.

Se ha conseguido dar a conocer los contenidos de los órdenes de la ayuda y su aplicación en los cuidados de enfermería.

-Favorecer las relaciones y vínculos entre los alumnos desde la pertenencia al sistema.

Este objetivo se ha cumplido con un alto nivel de satisfacción por parte de los alumnos/as.

-Dar a conocer a los alumnos/as los órdenes del amor para mirar su sistema familiar y descubrir su pertenencia a él.

Se cubrió este objetivo con un alto grado de satisfacción de los alumnos/as.

Para finalizar las conclusiones, se retoma el objetivo de esta investigación que fue evaluar el impacto que la propuesta educativa de la asignatura "Afrontamiento a la muerte: intervención interdisciplinar" tiene sobre los estudiantes de enfermería.

-La intervención docente de la asignatura, ha tenido un alto impacto en los alumnos/as en dos dimensiones importantes para sus vidas. Por un lado, aumentando sus competencias académicas como futuros profesionales $\mathrm{y}$, por otro, adquiriendo habilidades y actitudes positivas sobre el final de la vida muerte y duelo, integrando la filosofía de vida "ayudar a morir implica aprender a vivir", sustituyendo la visión de rechazo y miedo a la muerte por la visión de la aceptación de la vida tal cual es.

\section{Referencias bibliográficas}

1. Palomar MC. Introducción a los Cuidados Paliativos. En: Pérez E, Gómez, Bennasar M. Fundamentos de los Cuidados Paliativos. España: Fuden; 2008.p35-63.

2. Chan M. Cuidados Paliativos. Ginebra: Organización Mundial de la Salud. [Internet].2014[citado 8 Mar de 2015]. Disponible en: http://www.who.int/cancer/palliative/es/

3. Instituto Nacional del Cáncer (NCI). Cuidados Paliativos durante el Cáncer. [Internet].2010 [citado Oct 2017]. Disponible en: www.cancer.gov/espanol/cancer/canceravanzado/opciones-de-cuidado/hoja-informativa-cuidadospaliativosinformativas/apoyorecursos/ cuidados-paliativos

4. De Oliveira Cruz RA, Cartaxo Gomes de Arruda AJ, Glenda Agra G, Lopes Costa MM, De Medeiros Nóbrega VK. Reflexiones sobre los cuidados paliativos en el contexto de la formación en enfermería. J Nurs UFPE. 2016; 10(8):1-7.

5. Palomar Gallardo MC. Proyecto socio-sanitario formativo para familiares de personas en situación de enfermedad avanzada o terminal. En: XX Congreso Juventud, Familia y Sociedad. Construyendo una Ciudadanía Solidara: 23,24 y 25 de noviembre de 2005, Huelva: Organizado por el Centro de Inserción Sociolaboral Valdoco.

6. Palomar Gallardo MC, Arana Álvarez R, González Rodríguez A, Merino Navarro D, García Padilla.FM. Los Cuidados Paliativos en el Domicilio, una Experiencia de Formación Pregrado Enmarcada en el proyecto socio-sanitario del distrito V de Huelva. En: VII congreso de la Asociación Andaluza de Enfermería Comunitaria (ASANEC): 14 y 15 de junio 2007, Úbeda Jaén. Reconocido de interés Científico Sanitario por la Consejería de la Junta de Andalucía. 
7. Palomar Gallardo MC, Merino Navarro D, González Rodríguez A y Arana R. Taller de Bioenergetica: La promoción del bienestar de personas enfermas y familiares en el contexto de un proyecto de intervención comunitaria. En: VII congreso de la Asociación Andaluza de Enfermería Comunitaria (ASANEC):12 y 13 de junio 2008, Cádiz. Reconocido de interés Científico Sanitario por la Consejería de la Junta de Andalucía.

8. Olvera, A. Pedagogía Sistémica. Pedagogía del Siglo XXI. El éxito es tu historia. México: Grupo CUDEC; 2009.

9. Olvera, A. Traveset, M. Parellada C. Raíces, vínculos y alas. Una Pedagogía de la abundancia. México: Grupo CUDEC; 2012.

10. Hellinger B. Los órdenes de la ayuda. Buenos Aires: Alma Lepik; 2006.

11. Hellinger B. Órdenes del amor. Barcelona: Herder; 2008.

12. Garriga J. Vivir en el alma. España: Rigden Institut Gestalt; 2008.

13. Ortega Galán AM. El proceso de la muerte en el ámbito formal de los cuidados: un estudio cualitativo desde la perspectiva profesional sanitaria de Huelva. Tesis doctoral. Universidad de Huelva [Internet] 2012. [citado. 1 Mar 2015]. Disponible en: http://rabida.uhu.es/dspace/handle/10272/6044

14. Palomar MC, Ortega A, Ibáñez O. Últimos días. El proceso de Morir. Disponible en: Pérez E, y Medina FJ. Aspectos Psicosociales en Cuidados Paliativos. Madrid: Ed Enfoediciones; 2010.p.205-249.

15. Sanz Ortiz J, López Imedio E. Enfermería en cuidados paliativos. Madrid: Panamericana; 1998.

16. Portero de la Cruz S, Cebrino Cruz J. Afrontamiento de los estudiantes de Enfermería ante la muerte. Metas Enferm 2017; 20(4): 4-8.

17. Prado RT, Leite JL, Silva IR, Silva LJ, Castro EAB. The process of dying/death: intervening conditions to the nursing care management. Rev Bras Enferm [Internet]. 2018;71(4):200513. http://dx.doi.org/10.1590/0034-7167-2017-0173

18. Pérez ME, Cibanal LJ. Impacto psicosocial en enfermeras que brindan cuidados en fase terminal. Rev Cuid. 2016; 7(1): 1210-8.http://dx.doi.org/10.15649/cuidarte.v7i1.295

19. Stochero HM, Nietsche EA, Salbego C, Pivetta A, Schwertner MVE, Fettermann FA \& Rodrigues de Lima MG. Sentimentos e dificuldades no enfrentamento do processo de morrer e morte por graduandos de enfermagem. Aquichan. 2016; 16(2): 219-229.

20. Silva Oliveira E, Agra G, Formiga Morais M, Pereira Feitosa I, André Gouveia BL, Lopes Costa MM. El proceso de muerte y morir en la percepción de académicos de enfermería. Rv J Nurs UFPE. 2016;10 (5):1709-16.

21. Alonso, J P, La construcción del morir como un proceso: la gestión del personal de salud en el final de la vida. Universitas Humanística [Internet] 2012. [Fecha de consulta: 28 de noviembre de 2018] Disponible en: $\leq$ http://www.redalyc.org/articulo.oa?id=79125420006 $>$ ISSB 0120-4807

22. Lima R, Bergold LB, Souza JDF, Barbosa GS, Ferreira MA. Death education: sensibility for caregiving. Rev Bras Enferm. 2018;71(4):1779-84.

23. Kubler R. Sobre la muerte y los moribundos. México: Grijalbo; 1996.

24. De Hennezel M. La muerte íntima. Barcelona: Plaza y Janés; 1996.

25. Álvarez Gayou JL. Cómo hacer investigación cualitativa. México: Paidós Educador; 2003. 
26. Meleis AL. Theoretical nursing: development and progress. $3^{\text {a }}$ ed. Philadelfia: J.B. Lippincott; 1997.

27. Marriner-Tomey A. Modelos y teorías de enfermería. Madrid: Mosby/Doyma; 1994.

28. Hernández Conesa JM, Moral de Calatrava P, Esteban-Albert M. Fundamentos de la Enfermería, Teorías y Modelos. 2a ed. Madrid. McGraw-Hill: Interamericana; 2003.

29. Guillaumet M, Amorós G, Ramos A, Campillo B, Martínez Momblan MA. La narrativa como estrategia didáctica para una aproximación al proceso de la muerte. Rv enferm global [Internet]. 2018; [marz 2019)]; 14(49):197-12. http://dx.doi.org/10.6018/eglobal.17.1.260491 30. San Rafael Gutiérrez S, Siles González J Solano Ruiz C. El diario del estudiante de enfermería en la práctica clínica frente a los diarios realizados en otras disciplinas. Una revisión integradora. Aquichan [Internet] 2014. [20 Nov 2018]; 14(3):403-416 Disponible en: http://aquichan.unisabana.edu.co/index.php/aquichan/article/view/2218/pdf

31. Medina JL. La pedagogía del cuidado: saberes y prácticas en la formación Universitaria en Enfermería. Barcelona: Laertes; 1999.

Cómo citar: Palomar Gallardo M.C., Romero Palomar M., Pérez García E., Romero Palomar M.I. Educar para la vida y la muerte a los estudiantes de Enfermería: “del miedo al amor". Enfermería: Cuidados Humanizados. 2020; 9(1): 65-81. Doi: https://doi.org/10.22235/ech.v9i1.2167

Contribución de los autores: a) Concepción y diseño del trabajo, b) Adquisición de datos, c) Análisis e interpretación de datos, d) Redacción del manuscrito, e) Revisión crítica del manuscrito. MCPG ha contribuido en a,b,c,d; MRP en c; EPG en e; MIRP en d.

Editora científica responsable: Dra. Natalie Figueredo 